\title{
Fatores associados às perdas dentárias entre adultos em áreas rurais do estado de Pernambuco, Brasil
}

\author{
Factors associated with tooth loss among adults in rural areas in the \\ state of Pernambuco, Brazil
}

Patrícia Morgana Hordonho Santillo ${ }^{1}$

Estela Santos Gusmão ${ }^{2}$

Cristiano Moura ${ }^{3}$

Renata de Souza Coelho Soares ${ }^{4}$

Renata Cimões ${ }^{5}$

\footnotetext{
${ }^{1}$ Departamento de Ciências Básicas, Faculdade de Odontologia do Recife. R. Artur Coutinho 143, Santo Amaro. 50.100-280 Recife PE Brasil. phsantillo@hotmail.com ${ }^{2}$ Faculdade de Odontologia de Pernambuco, Universidade de Pernambuco.

${ }^{3}$ Universidade Federal de Campina Grande.

${ }^{4}$ Universidade Federal da Paraíba.

${ }^{5}$ Universidade Federal de Pernambuco.
}

Abstract The scope of this study was to estimate the prevalence of tooth loss and associated factors among Brazilian adults aged 20 to 59 years in rural areas in the state Pernambuco, Brazil. A crosssectional study was conducted on a random sample of 568 participants. The number of lost teeth (d" 12 and > 12) was the outcome investigated. The independent variables were as follows: sociodemographic characteristics, subjective oral health conditions, impact of oral health on quality of life using the short version of the Oral Health Impact Profile (OHIP-14), and the use of dental services. Crude and adjusted prevalence ratios were estimated using a Poisson regression model. The prevalence of subjects with at least one tooth lost was 91.4\%. Tooth loss was strongly associated with age group, self-perceived oral health and oral health impact on quality of life. The high prevalence of tooth loss among Brazilian adults in rural areas shows a need for a reorientation of public dental services aimed at this population group.

Key words Oral health, Tooth loss, Adult, Rural population, Quality of life, Cross-sectional studies
Resumo O objetivo deste estudo foi estimar a prevalência de perdas dentárias e fatores associados em adultos de 20 a 59 anos de idade em áreas rurais do estado de Pernambuco, Brasil. Foi realizado um estudo transversal com uma amostra aleatória de 568 indivíduos. O número de perdas dentárias $(\leq 12 e>12)$ foi o desfecho investigado. As variáveis independentes foram: características sociodemográficas, condições subjetivas relacionadas à saúde bucal, impacto da saúde bucal na qualidade de vida através do Oral Health Impact Profile na sua versão reduzida (OHIP-14) e utilização de serviços odontológicos. Foram estimadas as razões de prevalência bruta e ajustada através de regressão de Poisson. A prevalência de pelo menos uma perda dentária entre os indivíduos da amostra foi de 91,4\%. Perdas dentárias foram fortemente associadas à faixa etária, à autopercepção da saúde bucal e ao impacto da saúde bucal na qualidade de vida. A alta prevalência de perdas dentárias em adultos em áreas rurais confirma a necessidade de haver uma reorientação dos serviços públicos odontológicos voltados para esse contingente populacional.

Palavras-chave Saúde bucal, Perda de dente, Adulto, População rural, Qualidade de vida, Estudos transversais 


\section{Introdução}

A perda dos elementos dentários interfere diretamente na vida diária dos indivíduos, gerando uma desestabilização do sistema estomatognático em termos de redução da capacidade mastigatória e fonação, além de dificultar e limitar o consumo de diversos alimentos ${ }^{1}$. No Brasil, a alta proporção de indivíduos com perda dentária, bem como a possibilidade de controle desse agravo e seu impacto na vida diária das pessoas afetadas, ainda representa um desafio para a saúde pública².

Em se tratando das condições de saúde bucal na população adulta rural brasileira, evidenciase a realização de poucos estudos ${ }^{2-4}$ com o objetivo de traçar o perfil epidemiológico dessa população. Sabe-se, no entanto, que o seu acesso aos serviços públicos de forma geral, hospitais e unidades de saúde, pode ser difícil em razão da distância das propriedades e da precariedade dos serviços de transporte ${ }^{5}$. Além disso, as áreas rurais brasileiras muitas vezes apresentam piores indicadores de renda, saneamento básico e níveis de escolaridade que as respectivas áreas urbanas, tornando-as importantes polos de concentração para os agravos à saúde bucal ${ }^{6}$.

Embora a perda dentária não seja o indicador mais utilizado na pesquisa odontológica, alguns autores advogam a utilização desta "anomalia" como um importante desfecho clínico, indicador de saúde bucal ${ }^{7}$. No entanto, a simples avaliação das condições de saúde bucal por meio de critérios clínicos normativos pode não ser suficiente para elucidar a percepção do indivíduo acerca dessa sua condição, bem como da interferência desta na sua vida diária ${ }^{8}$. Nesse sentido, diversos indicadores sociodentais foram desenvolvidos para avaliar o impacto subjetivo das condições de saúde bucal na qualidade de vida de indivíduos e populações ${ }^{9,10}$. Entre esses indicadores está o Oral Health Impact Profile (OHIP$49)^{11}$, que é bastante difundido e empregado por sua facilidade de uso, principalmente na sua versão reduzida (OHIP-14) ${ }^{12}$. Esse instrumento foi traduzido, adaptado transculturalmente e validado para populações de adultos e idosos em diversas línguas, inclusive para o português, tanto em populações urbanas ${ }^{13}$ como em rurais ${ }^{14}$.

Em populações social e economicamente desfavorecidas, a prevalência de doenças passíveis de prevenção geralmente se apresenta mais elevada quando comparada a localidades mais desenvolvidas. Nesse sentido, populações rurais, vivendo isoladas dos centros urbanos e com limitado acesso aos serviços de saúde bucal, po- dem perceber os impactos da perda dentária na vida diária de forma diferenciada ${ }^{15}$.

O objetivo deste estudo foi estimar a prevalência de perdas dentárias em indivíduos adultos residentes em áreas de assentamento rural no Estado de Pernambuco, bem como investigar associações desse agravo a condições sociodemográficas, à autopercepção, ao impacto na qualidade de vida e à utilização de serviços odontológicos.

\section{Métodos}

Trata-se de um estudo transversal, constituído por uma população de referência de 4.235 indivíduos na faixa etária de 20 a 59 anos, residentes em 48 áreas de assentamento rural, realizado entre outubro de 2010 e janeiro de 2011. As 48 áreas envolvidas no estudo compõem o Assentamento Rural Governador Miguel Arraes, que abrange os municípios de Água Preta, Catende, Jaqueira, Palmares e Xexéu, pertencentes à III GERES (Gerência Regional de Saúde) - Palmares (PE), localizados na mesorregião da zona da mata do Estado de Pernambuco, com uma população geral de aproximadamente 24.000 habitantes.

O projeto de pesquisa foi aprovado pelo Comitê de Ética em Pesquisa da Universidade de Pernambuco - CEP/UPE, com registro no Sisnep (Sistema Nacional de Informação sobre Ética em Pesquisa envolvendo Seres Humanos). O estudo foi conduzido de acordo com os princípios da Declaração de Helsinki, tendo os participantes assinado o Termo de Consentimento Livre e Esclarecido (TCLE).

A amostra, do tipo probabilística, foi calculada com base nos seguintes parâmetros: prevalência de perdas dentárias entre indivíduos adultos de $50,0 \%$, poder de $80,0 \%$ para detectar uma diferença mínima de 20,0\% entre grupos, erropadrão de 5,0\% e intervalo de confiança de 95,0\% (IC95\%). A amostra final, acrescida de um fator de correção (efeito de agregação) de 1,2 e de 20,0\% de perdas, correspondeu a 691 indivíduos.

Adotou-se a amostragem por conglomerado em duplo estágio. Primeiramente, as famílias foram sorteadas por meio de listas fornecidas pelo sistema de informações de projetos de reforma agrária do Instituto Nacional de Colonização e Reforma Agrária (INCRA) - Regional Pernambuco. Posteriormente realizou-se o sorteio dos indivíduos que participaram do estudo.

Os critérios de inclusão foram: ter idade igual ou superior a 20 anos; ser morador das áreas do 
Assentamento Rural Miguel Arraes; e estar presente na residência nos dias pré-agendados para a realização do estudo. Foram excluídos da amostra os indivíduos que apresentavam algum impedimento físico e/ou mental para responder às perguntas da entrevista e/ou participar do exame físico.

O procedimento de calibração interexaminador foi realizado através do exame de duas sequências de 12 pacientes cada, os quais apresentavam as mais variadas condições clínicas, a fim de contemplar todas as condições dos critérios de diagnóstico preconizados pela Organização Mundial da Saúde (OMS) ${ }^{16}$. A concordância verificada pelo teste kappa $(\mathrm{k})$ indicou $\mathrm{k}=0,82$ (interexaminador). Durante a coleta de dados propriamente dita, cerca de $10,0 \%$ da amostra foi reexaminada (um a cada 10 examinados era sorteado), conferindo uma concordância intraexaminador $\mathrm{k}=0,91$.

Previamente à coleta de dados, realizou-se um estudo piloto envolvendo 30 indivíduos randomicamente selecionados, os quais não fizeram parte da pesquisa principal. Nesse estudo piloto foram testados o questionário padronizado e o formulário específico para a realização do exame físico.

As entrevistas e os exames físicos foram realizados nos domicílios dos participantes por uma equipe de campo (uma cirurgiã-dentista e uma anotadora), sob luz natural e por intermédio de inspeção visual e tátil, com o auxílio de espelhos bucais planos e sondas periodontais do tipo ballpoint, ambos esterilizados. A equipe estava devidamente paramentada de acordo com as normas de controle de infecção (utilização de gorros, luvas e máscaras descartáveis).

As variáveis independentes exploradas no presente estudo foram agrupadas em três blocos. No bloco 1 foram incluídas as variáveis sociodemográficas (sexo, faixa etária, cor da pele, escolaridade e renda familiar). A variável sexo foi categorizada em masculino e feminino e a faixa etária em 20-29, 30-39, 40-49 e 50-59 anos. A cor da pele foi coletada segundo as categorias autorreferidas utilizadas nos recenseamentos do Instituto Brasileiro de Geografia e Estatística (IBGE): brancos; negros; pardos; amarelos; indígenas. A escolaridade foi categorizada por anos de estudos em 0 anos (analfabeto), 1-8 anos e 9 ou mais anos. A renda familiar em salários mínimos, cujo valor de referência era de $\mathrm{R} \$ 510,00$, foi dicotomizada em inferior a um salário mínimo e igual ou superior a um salário mínimo. As variáveis do bloco 2 foram as seguintes: autopercepção da saúde bucal (ótima/boa; regular; ruim/péssima); autopercepção da dor de origem dental nos últimos seis meses (nenhuma dor; pouca dor; média/muita dor); autopercepção da necessidade de tratamento odontológico (sim; não); e o impacto da saúde bucal na qualidade de vida, mensurada por meio do OHIP- $14^{12}$, na sua forma simplificada. O questionário constitui-se de 14 perguntas, duas para cada uma das sete dimensões do instrumento: limitação funcional, dor física, desconforto psicológico, incapacidade física, incapacidade psicológica, incapacidade social e desvantagem. Utilizou-se como padrão de resposta às perguntas uma escala de Likert com os seguintes padrões: nunca; raramente; às vezes; repetidamente; sempre. Dessa forma, para fins de análise, os padrões de respostas para os sete domínios do questionário supracitado foram dicotomizados em: com impacto (repetidamente) sempre) e sem impacto (nunca/raramente/às vezes). O bloco 3 foi constituído por variáveis relacionadas à utilização de serviços odontológicos: tipo de serviço utilizado (público; privado); tempo decorrido após a última visita ao dentista, em anos $(<1 ; 1-2 ; \geq 3)$; motivo da visita ao dentista (consulta de rotina; dor; problemas odontológicos); avaliação do atendimento odontológico (positiva; negativa); e se recebeu informações sobre como evitar problemas bucais ( sim; não).

A variável dependente estudada foi a ocorrência de perdas dentárias, obtida a partir dos códigos 4 (perda dentária decorrente de cárie) e 5 (perda dentária decorrente de outras razões) do índice CPO-D (número de dentes cariados, perdidos e restaurados) para o diagnóstico da coroa de cada um dos 32 espaços dentários examinados por indivíduo, conforme os critérios de diagnóstico da $\mathrm{OMS}^{16}$. Posteriormente, para fins de análise, essa variável foi dicotomizada com base na média de dentes perdidos entre os indivíduos da amostra, resultando no ponto de corte de perda de até 12 dentes $(\leq 12)$ e perda superior a 12 dentes $(>12)$.

No modelo teórico hierárquico de determinação das perdas dentárias adotado neste estudo, as variáveis sociodemográficas (bloco 1) foram situadas na posição mais distal em relação ao desfecho. Admite-se que essas variáveis influenciam as condições subjetivas relacionadas à saúde bucal (bloco 2), bem como o padrão e a utilização de serviços odontológicos (bloco 3). As variáveis dos blocos 2 e 3, por sua vez, guardam entre si uma relação direta e de reciprocidade, influenciando sobremaneira a variável dependente da presente pesquisa. 
Para avaliar a existência de associação entre o desfecho e as demais variáveis independentes, foi realizada análise bivariada através do teste quiquadrado, adotando-se um nível de significância de 5\% (p < 0,05) e IC95\%.

Posteriormente, para estimar as razões de prevalência bruta e ajustada e seus respectivos IC95\% e valor de p (através do teste de Wald), foi realizada a regressão de Poisson com estimação de variância robusta para as variáveis que obtiveram $\mathrm{p} \leq 0,250$ na análise bivariada. Dessa forma, a modelagem estatística seguiu o modelo teórico hierárquico de determinação das perdas dentárias supracitado. As variáveis do bloco 1 (sociodemográficas) foram ajustadas entre si. As variáveis relacionadas às condições subjetivas em saúde bucal (bloco 2) foram ajustadas entre si e pelas variáveis do bloco 1 . Posteriormente, as variáveis relativas à utilização de serviços odontológicos (bloco 3) foram ajustadas entre si e pelas variáveis dos níveis superiores (blocos 1 e 2).

Os dados do presente estudo foram duplamente digitados e validados utilizando-se o software Epi Info 6.04 (Centers for Disease Control and Prevention, Atlanta, Estados Unidos). Para a análise estatística utilizou-se o software SPSS 13.0 (SPSS Inc., Chicago, Estados Unidos), bem como o software Stata/SE 10.0 (Stata Corp., College Station, Estados Unidos).

\section{Resultados}

A amostra total $(\mathrm{n}=568)$, que correspondeu a uma taxa de seguimento de $82,2 \%$, foi composta por $72,1 \%$ de mulheres $(n=410)$ e $27,8 \%(n=$ 158) de homens. A média de idade na amostra foi de 32,8 anos (mediana $=31,0$ e desvio padrão $=10,7)$. Em relação à cor da pele, 90,5\% ( $\mathrm{n}=$ 514) dos indivíduos autodeclararam-se negros ou pardos. Não houve referência às categorias amarelo ou indígena.

Observou-se que 96,6\% ( $\mathrm{n}=549)$ dos indivíduos entrevistados tinham renda familiar igual ou superior a um salário mínimo. Quanto ao grau de instrução, 73,2\% $(\mathrm{n}=416)$ dos indivíduos tinham entre 1 e 8 anos de estudo, e 18,5\% (n =105) informaram ser analfabetos.

O CPO-D médio entre os indivíduos foi de 15,9 ( mediana $=15,0$ e desvio padrão $=8,8)$, com uma média de dentes perdidos de 11,7 (mediana $=10,0$ e desvio padrão $=9,3$ ). Com base nessa média foi determinado o valor do ponto de corte para perdas dentárias em $\leq 12$ ou $>12$ dentes perdidos. A prevalência de pelo menos uma perda dentária entre os indivíduos da amostra foi de 91,4\%.
Na Tabela 1, pode-se verificar a associação entre a perda dentária e as variáveis sociodemográficas (bloco 1). Observa-se que a faixa etária foi a variável que apresentou maior razão de prevalência (RP), ou seja, as pessoas com idade entre 50 e 59 anos apresentavam aproximadamente 10 vezes maior prevalência de perda dentária superior a 12 dentes, quando comparadas àquelas que estavam na faixa etária de 20 a 29 anos. A escolaridade foi outra variável fortemente associada à perda dentária. As pessoas analfabetas apresentaram 4,6 vezes maior prevalência de perda dentária superior a 12 dentes, quando comparadas àquelas com escolaridade igual ou superior a nove anos de estudo.

As condições subjetivas relacionadas à saúde bucal mostraram-se associadas ao número de dentes perdidos, conforme Tabela 1. As pessoas que autoavaliaram sua saúde bucal como ruim ou péssima apresentaram 1,14 vezes maior prevalência de perda dentária em relação àquelas que consideraram sua saúde bucal como ótima ou boa. Observou-se, ainda, que o impacto da perda dentária foi 51\% maior nas pessoas com perda superior a 12 dentes do que naquelas com perda de até 12 dentes.

No que diz respeito às variáveis relacionadas à utilização de serviços odontológicos, verificouse que os adultos que relataram um tempo decorrido após a sua última consulta com o dentista de 1 a 2 anos ou igual ou superior a três anos apresentavam maior prevalência de perda dentária, como pode ser observado na Tabela 1 .

A Tabela 2 apresenta os modelos de análise multivariada seguindo o modelo teórico de determinação. Após terem sido realizados os devidos ajustes, a perda dentária manteve-se fortemente associada à faixa etária, à autopercepção da saúde bucal e ao impacto na qualidade de vida relacionado à saúde bucal.

\section{Discussão}

Estudar as populações de uma área rural brasileira evidenciou informações importantes no tocante ao conhecimento da sua saúde bucal, tanto pela falta de dados existentes como pelas características da localização estudada. São áreas remotas, de difícil acesso e que se encontram dispersas, sendo escasso o transporte, a água de abastecimento, o saneamento básico e os serviços públicos, apresentando, dessa forma, elevado grau de necessidade de saúde.

Além do exposto, é relevante o estudo pelo fato de se tratar de uma pesquisa realizada em 
Tabela 1. Associação entre perdas dentárias e variáveis sociodemográficas, autopercepção e impacto da saúde bucal e de utilização de serviços odontológicos entre adultos em áreas rurais. Pernambuco, Brasil, 2010/ 2011.

\begin{tabular}{|c|c|c|c|c|c|}
\hline \multirow[b]{2}{*}{ Variável } & \multicolumn{2}{|c|}{$\begin{array}{l}\text { Número de dentes } \\
\text { perdidos }\end{array}$} & \multirow[b]{2}{*}{$\begin{array}{l}\text { Total } \\
\text { n }(\%)\end{array}$} & \multirow[b]{2}{*}{ RP (IC95\%) } & \multirow[b]{2}{*}{$\begin{array}{l}\text { Valor } \\
\text { de } p^{\star}\end{array}$} \\
\hline & $\begin{array}{l}>12 \\
\text { n }(\%)\end{array}$ & $\begin{array}{l}\leq 12 \\
\mathrm{n}(\%)\end{array}$ & & & \\
\hline \multicolumn{6}{|l|}{ Bloco 1} \\
\hline \multicolumn{6}{|l|}{ Sexo } \\
\hline Masculino & $61(38,6)$ & $97(61,4)$ & $158(27,8)$ & 1,00 & 0,644 \\
\hline Feminino & $167(40,7)$ & $243(59,3)$ & $410(72,1)$ & $1,06(0,84-1,33)$ & \\
\hline \multicolumn{6}{|l|}{ Faixa etária (anos) } \\
\hline $20-29$ & $23(8,9)$ & $235(91,1)$ & $258(45,4)$ & 1,00 & $<0,001$ \\
\hline $30-39$ & $80(50,6)$ & $78(49,4)$ & $158(27,8)$ & $5,68(3,73-8,64)$ & \\
\hline $40-49$ & $76(78,4)$ & $21(21,6)$ & $97(17,1)$ & $8,79(5,87-13,16)$ & \\
\hline $50-59$ & $49(89,1)$ & $6(10,9)$ & $55(9,7)$ & $9,99(6,69-14,92)$ & \\
\hline \multicolumn{6}{|l|}{ Cor/Raça } \\
\hline Branco & $14(25,9)$ & $40(74,1)$ & $54(9,5)$ & 1,00 & 0,025 \\
\hline Negro/pardo & $214(41,6)$ & $300(58,4)$ & $514(90,5)$ & $1,61(1,01-2,55)$ & \\
\hline \multicolumn{6}{|l|}{ Escolaridade (anos) } \\
\hline$\geq 9$ & $7(14,9)$ & $40(85,1)$ & $47(8,3)$ & 1,00 & $<0,001$ \\
\hline $1-8$ & $148(35,6)$ & $268(64,4)$ & $416(73,2)$ & $2,39(1,19-4,79)$ & \\
\hline 0 (analfabeto) & $73(69,5)$ & $32(30,5)$ & $105(18,5)$ & $4,67(2,33-9,35)$ & \\
\hline \multicolumn{6}{|l|}{ Renda familiar ${ }^{* *}$} \\
\hline 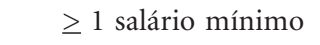 & $221(40,3)$ & $328(59,7)$ & $549(96,6)$ & 1,00 & 0,765 \\
\hline < 1 salário mínimo & $7(36,8)$ & $12(63,2)$ & $19(3,4)$ & $0,92(0,50-1,66)$ & \\
\hline \multicolumn{6}{|l|}{ Bloco 2} \\
\hline \multicolumn{6}{|l|}{$\begin{array}{l}\text { Autopercepção da saúde } \\
\text { bucal }\end{array}$} \\
\hline Ótima/boa & $70(42,7)$ & $94(57,3)$ & $164(29,5)$ & 1,00 & $<0,001$ \\
\hline Regular & $51(27,9)$ & $132(72,1)$ & $183(32,8)$ & $0,65(0,49-0,88)$ & \\
\hline Ruim/péssima & $102(48,6)$ & $108(51,4)$ & $210(37,7)$ & $1,14(0,91-1,43)$ & \\
\hline \multicolumn{6}{|c|}{$\begin{array}{l}\text { Autopercepção da dor de } \\
\text { dente }\end{array}$} \\
\hline Nenhuma dor & $141(42,2)$ & $193(57,8)$ & $334(59,3)$ & 1,00 & 0,054 \\
\hline Pouca dor & $23(28,0)$ & $59(72,0)$ & $82(14,6)$ & $0,66(0,46-0,96)$ & \\
\hline Média/muita dor & $62(42,2)$ & $85(57,8)$ & $147(26,1)$ & $1,00(0,80-1,25)$ & \\
\hline \multicolumn{6}{|c|}{$\begin{array}{l}\text { Autopercepção da } \\
\text { necessidade de tratamento }\end{array}$} \\
\hline Sim & $178(37,6)$ & $295(62,4)$ & $473(86,30)$ & 1,00 & $<0,001$ \\
\hline Não & $45(60,0)$ & $30(40,0)$ & $75(13,7)$ & $1,59(1,28-1,98)$ & \\
\hline \multicolumn{6}{|l|}{ OHIP - 14} \\
\hline Sem impacto & $50(29,6)$ & $119(70,4)$ & $169(29,7)$ & 1,00 & $<0,001$ \\
\hline Com impacto & $178(44,6)$ & $221(55,4)$ & $399(70,3)$ & $1,51(1,17-1,95)$ & \\
\hline
\end{tabular}

continua

domicílios com uma amostra aleatória de adultos residentes em áreas de assentamento rural do nordeste brasileiro, localizadas no estado de Pernambuco. Observou-se uma alta prevalência de perda de pelo menos um elemento dentário entre os indivíduos da amostra, estando ela fortemente associada às faixas etárias maiores, a uma autopercepção negativa e a um impacto negativo da saúde bucal na qualidade de vida dessas pessoas.

No Brasil o contingente populacional rural representa 15,6\% (aproximadamente 30 milhões de pessoas) da população brasileira, segundo o 
Tabela 1. continuação

\begin{tabular}{|c|c|c|c|c|c|}
\hline \multirow[b]{2}{*}{ Variável } & \multicolumn{2}{|c|}{$\begin{array}{l}\text { Número de dentes } \\
\text { perdidos }\end{array}$} & \multirow[b]{2}{*}{$\begin{array}{l}\text { Total } \\
\text { n (\%) }\end{array}$} & \multirow[b]{2}{*}{ RP (IC95\%) } & \multirow[b]{2}{*}{$\begin{array}{l}\text { Valor } \\
\text { de } p^{*}\end{array}$} \\
\hline & $\begin{array}{c}>12 \\
\text { n (\%) }\end{array}$ & $\begin{array}{c}\leq 12 \\
\mathrm{n}(\%)\end{array}$ & & & \\
\hline \multicolumn{6}{|l|}{ Bloco 3} \\
\hline \multicolumn{6}{|l|}{ Tipo de serviço odontológico } \\
\hline Privado & $38(38,8)$ & $60(61,2)$ & $98(19,6)$ & 1,00 & 0,959 \\
\hline Público & $157(39,1)$ & $245(60,9)$ & $402(80,4)$ & $1,01(0,76-1,33)$ & \\
\hline \multicolumn{6}{|l|}{$\begin{array}{l}\text { Tempo decorrido após a } \\
\text { última visita ao dentista } \\
\text { (anos) }\end{array}$} \\
\hline$<1$ & $77(30,9)$ & $172(69,1)$ & $249(45,0)$ & 1,00 & $<0,001$ \\
\hline $1-2$ & $57(49,6)$ & $58(50,4)$ & $115(20,8)$ & $1,60(1,23-2,08)$ & \\
\hline$\geq 3$ & $92(48,7)$ & $97(51,3)$ & $189(34,2)$ & $1,57(1,24-1,99)$ & \\
\hline \multicolumn{6}{|l|}{ Motivo da visita ao dentista } \\
\hline Consulta de rotina & $14(43,8)$ & $18(56,2)$ & $32(5,8)$ & 1,00 & 0,359 \\
\hline Dor & $139(43,0)$ & $184(57,0)$ & $323(58,4)$ & $0,98(0,65-1,49)$ & \\
\hline Problemas odontológicos & $73(36,9)$ & $125(63,1)$ & $198(35,8)$ & $0,84(0,55-1,30)$ & \\
\hline \multicolumn{6}{|l|}{$\begin{array}{l}\text { Avaliação do atendimento } \\
\text { odontológico }\end{array}$} \\
\hline Positiva & $166(40,7)$ & $242(59,3)$ & $408(83,6)$ & 1,00 & 0,027 \\
\hline Negativa & $22(27,5)$ & $58(72,5)$ & $80(16,4)$ & $0,68(0,46-0,98)$ & \\
\hline \multicolumn{6}{|l|}{$\begin{array}{l}\text { Recebeu informações sobre } \\
\text { como evitar problemas }\end{array}$} \\
\hline bucais & $90(36,9)$ & $154(63,1)$ & $244(49,9)$ & 1,00 & 0,424 \\
\hline Sim & $99(40,4)$ & $146(59,6)$ & $245(50,1)$ & $1,10(0,88-1,37)$ & \\
\hline Não & & & & & \\
\hline
\end{tabular}

IC95\% = intervalo de confiança de 95\%; OHIP-14 = Oral Health Impact Profile, na sua versão reduzida contendo 14 perguntas; $\mathrm{RP}=$ razão de prevalência. ${ }^{*}$ Teste qui-quadrado. ${ }^{* *}$ Valor de referência do salário mínimo foi de $\mathrm{R} \$ 510,00$.

censo do IBGE de $2010^{17}$. No entanto, estabelecer comparações acerca das condições de saúde bucal na população rural brasileira torna-se difícil em virtude da grande ênfase dos estudos em populações urbanas. De uma maneira geral, na população rural investigada nesta pesquisa, as mulheres foram as que apresentaram maior número de perdas dentárias, seguidas dos indivíduos que tinham mais idade, daqueles que se autodeclararam pardos ou negros e dos que tinham renda familiar igual ou superior a um salário mínimo.

Sabe-se que as condições sociais dos indivíduos e a prática odontológica hegemônica, que considera a extração dos elementos dentários a solução para o alívio da dor em populações de baixo nível socioeconômico, exercem um importante papel na prevalência da perda dentária ${ }^{18}$. Em estudo proposto por Silva et al. ${ }^{15}$ e corroborado por Vargas e Paixão ${ }^{18}$, por meio de entrevistas abertas e semiestruturadas com pacientes adultos de um Centro de Saúde em Belo Hori- zonte, Minas Gerais, as causas das perdas dentárias mais comuns apontadas pelos pacientes foram a falta de informação e de condições financeiras para o tratamento. Em se tratando de populações rurais, além das causas sociais e econômicas, o acesso aos serviços de saúde geral e bu$\mathrm{cal}^{2}$, em virtude da distância geográfica, constitui um condicionante a mais para a perpetuação dessa prática de solução imediata aos problemas de saúde bucal.

Apesar da alta prevalência observada de dentes perdidos entre os indivíduos desta amostra, a média foi de 11,7 dentes, sendo este o critério utilizado para o ponto de corte estabelecido na variável dependente. Ressalta-se que nesse cálculo não existem garantias de mastigação e/ou estéticas suficientes, evidenciando, assim, uma possível limitação deste estudo. Ademais, a média supracitada foi superior à encontrada por Saliba et al. ${ }^{5}$, que ao avaliarem uma amostra de 473 indivíduos residentes na zona rural do municí- 
Tabela 2. Análise multivariada da associação entre perdas dentárias e variáveis sociodemográficas, autopercepção e impacto da saúde bucal e utilização de serviços odontológicos entre adultos em áreas rurais. Pernambuco, Brasil, 2010/2011.

\begin{tabular}{|c|c|c|c|}
\hline Variável & $\begin{array}{c}\text { Modelo 1 } \\
\text { RP (IC95\%) }\end{array}$ & $\begin{array}{c}\text { Modelo } 2 \\
\text { RP (IC95\%) }\end{array}$ & $\begin{array}{c}\text { Modelo } 3 \\
\text { RP (IC95\%) }\end{array}$ \\
\hline \multicolumn{4}{|l|}{ Bloco 1} \\
\hline \multicolumn{4}{|l|}{ Faixa etária (anos) } \\
\hline $20-29$ & 1,00 & 1,00 & \\
\hline $30-39$ & $5,68(3,73-8,64)$ & $5,37(3,46-8,34)$ & \\
\hline $40-49$ & $8,79(5,87-13,16)$ & $7,41(4,77-11,53)$ & \\
\hline $50-59$ & $9,99(6,69-14,92)$ & $8,39(5,32-13,23)$ & \\
\hline Valor de $\mathrm{p}^{*}$ & $<0,001$ & $<0,001$ & \\
\hline \multicolumn{4}{|l|}{ Cor/Raça } \\
\hline Branco & 1,00 & 1,00 & \\
\hline Negro/pardo & $1,61(1,01-2,55)$ & $0,98(0,66-1,48)$ & \\
\hline Valor de $\mathrm{p}^{*}$ & 0,025 & 0,944 & \\
\hline \multicolumn{4}{|l|}{ Escolaridade (anos) } \\
\hline$\geq 9$ & 1,00 & 1,00 & \\
\hline $1-8$ & $2,39(1,19-4,79)$ & $1,40(0,74-2,65)$ & \\
\hline 0 (analfabeto) & $4,67(2,33-9,35)$ & $1,41(0,76-2,63)$ & \\
\hline Valor de $\mathrm{p}^{*}$ & $<0,001$ & 0,552 & \\
\hline \multicolumn{4}{|l|}{ Bloco 2} \\
\hline \multicolumn{4}{|c|}{ Autopercepção da saúde bucal } \\
\hline Ótima/boa & 1,00 & 1,00 & \\
\hline Regular & $0,65(0,49-0,88)$ & $0,83(0,63-1,10)$ & \\
\hline Ruim/péssima & $1,14(0,91-1,43)$ & $1,21(0,96-1,51)$ & \\
\hline Valor de $\mathrm{p}^{*}$ & $<0,001$ & $<0,01$ & \\
\hline \multicolumn{4}{|c|}{ Autopercepção da dor de dente } \\
\hline Nenhuma dor & 1,00 & 1,00 & \\
\hline Pouca dor & $0,66(0,46-0,96)$ & $0,74(0,51-1,09)$ & \\
\hline Média/muita dor & $1,00(0,80-1,25)$ & $0,97(0,81-1,18)$ & \\
\hline Valor de $\mathrm{p}^{*}$ & 0,054 & 0,324 & \\
\hline \multicolumn{4}{|c|}{$\begin{array}{l}\text { Autopercepção da necessidade de } \\
\text { tratamento }\end{array}$} \\
\hline Sim & 1,00 & 1,00 & \\
\hline Não & $1,59(1,28-1,98)$ & $1,20(0,93-1,55)$ & \\
\hline Valor de $\mathrm{p}^{*}$ & $<0,001$ & 0,157 & \\
\hline \multicolumn{4}{|l|}{ OHIP -14} \\
\hline Sem impacto & 1,00 & 1,00 & \\
\hline Com impacto & $1,51(1,17-1,95)$ & $1.43(1,09-1,88)$ & \\
\hline Valor de $\mathrm{p}^{*}$ & $<0,001$ & $<0,001$ & \\
\hline \multicolumn{4}{|l|}{ Bloco 3} \\
\hline \multicolumn{4}{|c|}{$\begin{array}{l}\text { Tempo decorrido após a última visita ao } \\
\text { dentista (anos) }\end{array}$} \\
\hline$<1$ & 1,00 & & 1,00 \\
\hline $1-2$ & $1,60(1,23-2,08)$ & & $1,02(0,82-1,26)$ \\
\hline$\geq 3$ & $1,57(1,24-1,99)$ & & $0,87(0,69-1,10)$ \\
\hline Valor de $\mathrm{p}^{*}$ & $<0,001$ & & 0,369 \\
\hline \multicolumn{4}{|c|}{ Avaliação do atendimento odontológico } \\
\hline Positiva & 1,00 & & 1,00 \\
\hline Negativa & $0,68(0,46-0,98)$ & & $1,43(1,09-1,88)$ \\
\hline Valor de $\mathrm{p}^{*}$ & 0,027 & & 0,244 \\
\hline
\end{tabular}

IC95\% = intervalo de confiança de 95\%; OHIP-14 = Oral Health Impact Profile, na sua versão reduzida contendo 14 perguntas; $\mathrm{RP}=$ razão de prevalência. Modelo 1: valores brutos; Modelo 2: variáveis do bloco 1 ajustadas entre si e variáveis do bloco 2 ajustadas entre si e pelo bloco 1; Modelo 3: variáveis do bloco 3 ajustadas entre si e pelas variáveis dos blocos 1 e 2 . ${ }^{\star}$ Teste de heterogeneidade de Wald. 
pio de Gabriel Monteiro, São Paulo, encontram uma média de 8,2 perdas dentárias. Em estudo conduzido por Barbato et al. ${ }^{2}$, a partir de dados secundários oriundos do Estudo Epidemiológico Nacional (Projeto SB Brasil 2002-2003), foi encontrada uma mediana de 13 dentes perdidos entre adultos brasileiros residentes de áreas rurais, parâmetro superior ao encontrado nesta pesquisa, que foi de 10 dentes perdidos.

Nesse sentido, os autores acima referidos justificam que a perda dentária pode ser decorrente da infraestrutura dos serviços de saúde no meio rural, que seria inferior à oferecida nas zonas urbanas, normalmente dispondo de menor acesso às tecnologias e à qualificação profissional, com consequente restrição dos procedimentos odontológicos àqueles mutiladores. Em consonância com esse pensamento, Al Shammery et al. ${ }^{19}$, em estudo realizado na Arábia Saudita, observaram que a média de dentes perdidos era de $3,5 \mathrm{em}$ zonas rurais e 4,2 em zonas urbanas, índices considerados baixos em decorrência da moderna infraestrutura dos serviços de saúde disponibilizados naquele país. Esses resultados, de uma maneira geral, evidenciam que uma melhor estruturação dos serviços de saúde tornaria possível dinamizar o atendimento às populações que vivem em zonas rurais.

Os resultados do presente estudo reafirmam o modelo de estudo proposto por Barbato et al. ${ }^{2}$, no qual o meio rural condiciona menores níveis de escolaridade, estando a baixa escolaridade associada a maiores perdas dentárias. No entanto, após os ajustes para os fatores de confusão, a baixa escolaridade não permaneceu significativamente associada à perda dentária.

As pesquisas de Matos et al..$^{20}$, corroboradas por Skudutyte et al. ${ }^{21}$, mostraram que indivíduos com mais anos de estudo tinham mais chance de receber tratamento restaurador ou preventivo quando comparados aos de menor escolaridade, inferindo que o maior nível de instrução acarretaria mais informação, conhecimento e acesso aos serviços de saúde. Para esses autores a perda dentária estigmatiza a população pobre, reforçando as desigualdades entre as classes sociais.

Ainda neste estudo, os resultados evidenciados quanto à variável cor da pele mostraram que os indivíduos autodeclarados negros ou pardos apresentaram mais perdas dentárias quando comparados aos autodeclarados brancos. Porém, da mesma forma como o ocorrido com a variável escolaridade, ao serem realizados os ajustes, essa associação deixou de existir, contrariamente ao que ocorreu nos estudos de Guiotoku et al..$^{22}$,
Gilbert et al..$^{23}$, Barbato et al. ${ }^{2}$ e Sabbah et al. ${ }^{24}$, nos quais a perda dentária esteve associada à cor da pele mesmo após o ajuste de possíveis variáveis de confusão.

O mesmo também foi demonstrado nos estudos de Chor e Lima ${ }^{25}$ e Sabbah et al. ${ }^{24}$, os quais indicam que as evidências de piores indicadores de saúde entre negros e pardos constituem-se disparidades étnico-raciais no Brasil e podem estar associadas à genética, a fatores culturais, a fatores relacionados à discriminação e a aspectos multidimensionais. Diante dos achados desses estudos, essas questões levam a crer que, apesar da presente pesquisa não ter encontrado essa associação, esta população encontra-se em desvantagem social comparada a outras. É possível que fatores relacionados ao perfil de desenvolvimento humano, como os raciais, e outros como os anos de estudo tenham papel essencial na caracterização da vulnerabilidade de grupos populacionais a agravos em saúde bucal, podendo afetar as chances de ascensão e constituindo um aspecto importante na investigação de fatores sociais capazes de afetar a saúde. Contudo, nesta população esses fatores parecem não ter sido por si só os responsáveis pelas perdas ocorridas.

É provável que, da mesma forma como constatado nos dados do Projeto SB Brasil 2002$2003^{26}$, na área onde foi realizado o presente estudo, mesmo com a expansão dos serviços de saúde no Brasil, a extração dentária continue a ser o procedimento disponível para os indivíduos adultos. Isso pode estar ocorrendo por se tratar de municípios localizados em áreas rurais, com porte populacional menor e em condições mais desfavoráveis. Desse modo, embora os serviços de saúde estejam disponibilizando procedimentos de atenção básica, existe uma necessidade em relação à atenção secundária, como os tratamentos endodônticos e, na ausência destes, a exodontia tem sido utilizada como a primeira opção para o alívio da dor. Em virtude disso, a qualidade dos cuidados de saúde dispensados aos indivíduos participantes da pesquisa ainda é questionável, sobretudo em relação aos indivíduos com maior faixa etária.

Assim, é possível destacar que as perdas dentárias na população estudada podem ser entendidas como sequelas não só das doenças bucais, mas também do processo de exclusão social sofrido por esses indivíduos no decorrer da vida, as quais somente poderão ser minimizadas através de políticas sociais e econômicas capazes de reduzir o risco de doenças e de promover o acesso igualitário aos serviços de saúde. 
Em relação às condições subjetivas relacionadas à saúde bucal, observou-se que os indivíduos que autoavaliaram a sua saúde bucal como ruim ou péssima, os que não tinham sentido dor dente nos últimos seis meses e aqueles que perceberam a necessidade de tratamento odontológico apresentavam, em sua maioria, um maior número de dentes perdidos. Nesse sentido, Bezerra et al. ${ }^{27}$ afirmam que a percepção precária da própria saúde pode ser vista como resultado de sentimentos provocados pelo mal-estar, dor ou desconforto, em interação com fatores sociais, culturais, psicológicos e ambientais que modificam a maneira como a vida da pessoa é afetada pelo problema experimentado. Sendo assim, essa percepção deve ser analisada sob uma ótica multidimensional, levando em consideração os diferentes entendimentos individuais da saúde diante do contexto cultural e psicossocial existente.

Em se tratando de estudo transversal, considera-se a possibilidade de ocorrência do viés de causalidade reversa, que ocorre quando a aparente exposição é consequência do desfecho. Esse fato pode ter ocorrido quando avaliamos tais condições subjetivas em relação às perdas dentárias.

Dentro desse contexto está inserido o impacto da saúde bucal na qualidade de vida, aferido neste estudo por meio do indicador OHIP-14. De fato, entre as pessoas que apresentavam um maior número de dentes perdidos, a maioria manifestou interferência da saúde bucal na vida diária. No entanto, essa expressividade do impacto pode ter sido gerada em função do maior número de dentes perdidos (> 12 perdas), com repercussão direta nas dimensões avaliadas por tal indicador. Sendo assim, a perda dentária poder ser considerada como uma saída para o fracasso de um tratamento conservador anteriormente realizado ${ }^{28}$, e pode ser referenciada como um forte preditor de impacto que, além de causar danos funcionais, é capaz de desequilibrar a organização psíquica e social dos indivíduos ${ }^{29}$. Vale ressaltar uma limitação relacionada ao construto da análise no que se refere à categorização do OHIP, que considera como sem impacto "às vezes", do mesmo modo que "nunca" e "raramente". Isso pode ter comprometido os resultados apontados nesta pesquisa.

Em se tratando da utilização de serviços odontológicos, os usuários de serviços públicos que decorreram um intervalo de tempo igual ou superior a três anos desde a última consulta odontológica, que tiveram como principal motivo a dor de origem dental, que avaliaram de maneira positiva o atendimento odontológico e que rela- taram não ter recebido informações sobre prevenção em saúde bucal apresentaram maior número de dentes perdidos. Diante dessa configuração, algumas hipóteses emergem na tentativa de explicar tais situações: a carga da doença é muito grande e o serviço ofertado não está capacitado para absorver a demanda ou, na contramão do paradigma da promoção de saúde, o serviço de saúde extrai mais em função da sua baixa resolutividade e a única alternativa, em muitos casos, é o tratamento mutilador. Essas são lacunas que ainda precisam ser preenchidas, aprofundando o conhecimento acerca dos serviços de saúde prestados às populações rurais brasileiras.

\section{Conclusão}

É alta a prevalência de perdas dentárias entre adultos residentes em áreas de assentamento rural, o que pode estar reforçando as desigualdades entre as classes sociais. O número de dentes perdidos esteve associado, por meio da análise bruta, à faixa etária, à cor da pele, à escolaridade, à autopercepção da saúde bucal e da necessidade de tratamento e ao impacto da saúde bucal na qualidade de vida, bem como ao tempo decorrido após a última consulta odontológica e à avaliação do atendimento. No entanto, após terem sido realizados os devidos ajustes, seguindo o modelo hierárquico de determinação proposto pelo estudo, permaneceram no modelo final as associações às variáveis: faixa etária, autopercepção da saúde bucal e impacto na qualidade de vida relacionada à saúde bucal. Evidências científicas acerca do conhecimento das condições de saúde bucal da população rural brasileira precisam ser geradas a fim de subsidiar a reorientação dos serviços públicos odontológicos voltados para esse contingente populacional.

\section{Colaboradores}

PMH Santillo, C Moura, RS Coelho-Soares participaram da concepção e planejamento, introdução, interpretação dos dados, discussão e da revisão crítica do conteúdo. ES Gusmão e R Cimões contribuíram para a concepção e planejamento, na interpretação dos dados, revisão crítica do conteúdo e na aprovação da versão final do manuscrito. 


\section{Referências}

1. Lacerda JT, Castilho EA, Calvo MCM, Freitas SFT. Saúde bucal e desempenho diário de adultos em Chapecó, Santa Catarina, Brasil. Cad Saude Publica 2008; 24(8):1846-1858.

2. Barbato PR, Muller Nagano HC, Zanchet FN, Boing AF, Peres MA. Perdas dentárias e fatores sociais, demográficos e de serviços associados em adultos brasileiros: uma análise dos dados do Estudo Epidemiológico Nacional (Projeto SB Brasil 20022003). Cad Saude Publica 2007; 23(8):1803-1814.

3. Silva DD, Rihs LB, Sousa Mda L. Fatores associados à presença de dentes em adultos de São Paulo, Brasil. Cad Saude Publica 2009; 25(11):2407-2418.

4. Frazão P, Antunes JLF, Narvai PC. Perda dentária precoce em adultos de 35 a 44 anos de idade. Estado de São Paulo, Brasil, 1998. Rev Bras Epidemiol 2003; 6(1):49-57.

5. Saliba NA, Moimaz SA, Saliba O, Tiano AV. Perda dentária em uma população rural e as metas estabelecidas pela Organização Mundial de Saúde. Cien Saude Colet 2010; 15(Supl.1):1857-1864.

6. Mello TRC, Antunes JLF. Prevalência de cárie dentária em escolares da região rural de Itapetininga, São Paulo, Brasil. Cad Saude Publica 2004; 20(3):829835.

7. Hujoel PP, DeRouen TA. A survey of endpoint characteristics in periodontal clinical trials published 1988-1992, and implications for future studies. $J$ Clin Periodontol 1995; 22(5):397-407.

8. Bianco VC, Lopes ES, Borgato MH, Moura e Silva P, Marta SN. O impacto das condições bucais na qualidade de vida de pessoas com cinquenta ou mais anos de vida. Cien Saude Colet 2010; 15(4):21652172.

9. Cohen LK, Jago JD. Toward the formulation of sociodental indicators. Int J Health Serv 1976; 6(4):681698.

10. Locker D. Measuring oral health: a conceptual framework. Community Dent Health 1988; 5(1):3-18.

11. Slade GD, Spencer AJ. Development and evaluation of the Oral Health Impact Profile. Community Dent Health 1994; 11(1):3-11.

12. Slade GD. Derivation and validation of a shortform oral health impact profile. Community Dent Oral Epidemiol 1997; 25(4):284-290.

13. Oliveira BH, Nadanovsky P. Psychometric properties of the Brazilian version of the Oral Health Impact Profile-short form. Community Dent Oral Epidemiol 2005; 33(4):307-314.

14. Cohen-Carneiro F, Rebelo MA, Souza-Santos R, Ambrosano GM, Salino AV, Pontes DG. Psychometric properties of the OHIP-14 and prevalence and severity of oral health impacts in a rural riverine population in Amazonas State, Brazil. Cad Saude Publica 2010; 26(6):1122-1130.

15. Silva RH, Castro RF, Cunha DC, Almeida CT, Bastos JR, Camargo LM. Cárie dentária em população ribeirinha do Estado de Rondônia, Região Amazônica, Brasil, 2005/2006. Cad Saude Publica 2008; 24(10):2347-2353.

16. World Health Organization (WHO). Oral Health Surveys: Basic Methods. $4^{\text {th }}$ Edition. Geneva: WHO; 1997.
17. Instituto Brasileiro de Geografia e Estatística (IBGE). Censo Demográfico 2010. [site da Internet]. [acessado 2012 fev 01]. Disponível em: http://www.ibge. gov.br.

18. Vargas AMD, Paixão HH. Perda dentária e seu significado na qualidade de vida de adultos usuários de serviço público de saúde bucal do Centro de Saúde Boa Vista, em Belo Horizonte. Cien Saude Colet 2005; 10(4):1015-1024.

19. al Shammery A, el Backly M, Guile EE. Permanent tooth loss among adults and children in Saudi Arabia. Community Dent Health 1998; 15(4):277-280.

20. Matos DL, Lima-Costa MF, Guerra HL, Marcenes W. Projeto Bambuí: avaliação de serviços odontológicos privados, públicos e de sindicato. Rev Saude Publica 2002; 36(2):237-243.

21. Skudutyte R, Aleksejuniene J, Eriksen HM. Dental caries in adult Lithuanians. Acta Odontol Scand 2000; 58(4):143-147.

22. Guiotoku SK, Moysés ST, Moysés SJ, França BH, Bisinelli JC. Iniquidades raciais em saúde bucal no Brasil. Rev Panam Salud Publica 2012; 31(2):135141.

23. Gilbert GH, Duncan RP, Shelton BJ. Social determinants of tooth loss. Health Serv Res 2003; 38(6 Pt 2):1843-1863

24. Sabbah W, Tsakos G, Sheiham A, Watt RG. The effects of income and education on ethnic differences in oral health: a study in US adults. $J$ Epidemiol Community Health 2009; 63(7):516-520.

25. Chor D, Lima CR. Epidemiologic aspects of racial inequalities in health in Brazil. Cad Saude Publica 2005; 21(5):1586-1594.

26. Brasil. Departamento de Atenção Básica. Secretaria de Atenção à Saúde. Ministério da Saúde (MS). Projeto SB Brasil 2003. Condições de saúde bucal da população brasileira 2002-2003: resultados principais. Brasília: MS; 2004

27. Bezerra PC, Opitz SP, Koifman RJ, Muniz PT. Percepção de saúde e fatores associados em adultos: inquérito populacional em Rio Branco, Acre, Brasil, 2007-2008. Cad Saude Publica 2011; 27(12):24412451.

28. Mendonça TC. Mutilação dentária: concepções de trabalhadores rurais sobre a responsabilidade pela perda dentária. Cad Saude Publica 2001; 17(6):15451547.

29. Wolf SMR. O significado psicológico da perda dos dentes em sujeitos adultos. Rev Assoc Paul Cir Dent 1998; 52(4):307-316.

Artigo apresentado em 07/11/2012

Aprovado em 13/01/2012

Versão final apresentada em 25/01/2013 